\title{
Impact of Microsatellite Instability in Signet-Ring Cell and Mucinous Components in Patients With Colorectal Carcinoma
}

\author{
Ik Yong Kim \\ Department of Surgery, Yonsei University Wonju College of Medicine, Wonju Severance Christian Hospital, Wonju, Korea
}

See Article on Page 58-65

The disease pathogenesis of colorectal cancer (CRC) is influenced by genetic and epigenetic events that occur with tumor initiation and progression [1]. Diagnostic approaches have evolved from relying exclusively on clinical criteria to incorporating pathologic features such as mucinous and signet-ring cell (SRC) carcinomas, PCR-based microsatellite instability (MSI) testing, and immunohistochemistry for loss of DNA mismatch repair (MMR) component expression [2]. In addition, molecular testing is routinely performed in clinical practice for the selection of patients for targeted biological agents and is advocated for prognostic stratification. Efforts to classify CRC that integrate these molecular features have been developed, and efforts to validate and refine these genetic subtypes to include additional genomic features are ongoing [3].

Recently, new molecular subtypes of biological relevance that were associated with different patient outcomes were identified, and four consensus molecular subtypes (CMSs) with distinguishing characteristics based on many CRC datasets, as well as common features among several independent classification systems, were accepted [4]. These are designated as CMS1 to CMS4. CMS1 (microsatellite instability or MSI, immune) is found in $14 \%$ of all patients with CRC and is characterized by MSI, CpG island methylator phenotype (CIMP)-high hypermutation, $B R A F$ mutations, increased expressions of genes related to diffuse immune infiltration consisting mainly of TH1 and cytotoxic T cells, activation of

Correspondence to: Ik Yong Kim, M.D.

Division of Colorectal Surgery, Department of Surgery, Yonsei University, Wonju College of Medicine, 20 Ilsan-ro, Wonju 26426, Korea

Tel: +82-33-741-0573, Fax: +82-33-741-0574

E-mail: iykim@yonsei.ac.kr

(c) 2016 The Korean Society of Coloproctology

This is an open-access article distributed under the terms of the Creative Commons Attribution NonCommercial License (http://creativecommons.org/licenses/by-nc/4.0) which permits unrestricted noncommercial use, distribution, and reproduction in any medium, provided the original work is properly cited. pathways to evade the immune system, low somatic copy number alterations and a worse prognosis after relapse. CMS1 includes most MSI carcinomas with overexpression of DNA damage-repair proteins and impaired DNA mismatch repair ability. CMS1 is also frequently found in female patients with right-sided tumors with high histological grade.

In patients with CRC, those with high-MSI cancers (20\%) have been shown to have a better overall prognosis than patients with microsatellite stable (MSS) cancers. In patients with MSS cancers, histologic features are more commonly seen that are poorly differentiated, are mucinous, lack dirty necrosis, have increased intense tumor infiltrating lymphocytes (TIL cells), have a circumscribed/expansile growth pattern, and are histologically heterogeneous. For patients with CRC presenting with SRCs, mucinous components (MUCs) and poorly differentiation cells, the tumors are histologically not homogenous, the outcomes are not uniform, molecular features for carcinogenesis are different, and little is known about the prognostic significance of these component [5].

In 1,336 patients with CRC, higher proportions of SRCs and MUCs were both associated with proximal tumor location, MSIhigh and CIMP-high cancers, MLH1 promoter hyper-methylation, frequent $B R A F$ mutation, and higher LINE-1 methylation level [6]. As to CRC-specific mortality, two cohort studies showed higher mortality in patients with CRC presenting with SRCs (hazard ratio [HR], 1.16; 95\% confidence interval [CI], 1.09-1.24), but not MUCs (multivariate HR, 0.99; 95\% CI, 0.95-1.04). Those authors 6 also examined the impact of MSI status on the prognosis for patients with CRC presenting with both SRCs and MUCs. They found that the CRC-specific mortality for patients presenting with SRCs did not differ by MSI status. However, in the multivariate analysis, they found that a high-MSI mucinous carcinoma was associated with lower CRC-specific mortality than a MSS mucinous carcinoma. Therefore, they concluded that, compared to the MUC, SRCs were independent of other clinicopathological and tumor-related molecular characteristics.

The author of this study reported that patients with CRC presenting with MUCs, SRCs, and poorly differentiated cells are bet- 
ter stratified by survival when the MSI status of the tumor is considered [7]. However, the analysis had several limitations. Firstly, the size of each pathology group was small. Secondly, the frequencies of patients presenting with MUCs and MSI show geographical variations. Many reported frequencies of MUC are different between Asian (3\%-12\%) and Western (10\%-39\%) populations [3]. MSI is also more frequently found in Western patients. In stage III CRC, $11 \%-21 \%$ of Western patients present with MSI whereas $5 \%-8 \%$ of Asian patients do. Thus, a large nationwide study to survey the incidence of MSI in patients with CRC and to identify its impact on prognosis among patients presenting with distinct histologies, such as SRCs or MUCs and high-MSI cancer, is needed.

Theoretically, MMR deficiency causing many somatic mutations may produce 'non-self' immunogenic antigens or neoantigens, as shown by the expression of immune checkpoint ligands PD1, PD-LI, CTLA-4, LAG-3, and IDO., etc. Le et al. [8] actually reported significant responses of cancers with MSI to anti-PD-1 immune checkpoint inhibitors in patients whose cancer had not responded to conventional therapy. This phase II study using pembrolizumab showed that the MMR status of a tumor could be used to predict response to therapy. For patients with MMR-deficient cancer, the immune-related objective response rate and the progression-free survival rate were higher they were for patients without MMR deficiency [8]. This study opened a new window of research associating somatic hypermutation and neoepitope formation with response to immunotherapy.

Gene analysis has proven to be a new approach for judging the potential clinical benefit of immune checkpoint inhibitors [9], such as mutational landscape and mismatch-repair deficiency [10]. Further preclinical and clinical studies are necessary before this idea can be applied in clinical practice.

\section{CONFLICT OF INTEREST}

No potential conflict of interest relevant to this article was re- ported.

\section{REFERENCES}

1. Coppede F, Lopomo A, Spisni R, Migliore L. Genetic and epigenetic biomarkers for diagnosis, prognosis and treatment of colorectal cancer. World J Gastroenterol 2014;20:943-56.

2. Bosman FT, Carneiro F, Hruban RH, Theise ND. WHO classification of tumours of the digestive system. 4 th ed. Geneva: International Agency for Research on Cancer; 2010.

3. Kim JH, Kang GH. Molecular and prognostic heterogeneity of microsatellite-unstable colorectal cancer. World J Gastroenterol 2014;20:4230-43.

4. Guinney J, Dienstmann R, Wang X, de Reyniès A, Schlicker A, Soneson C, et al. The consensus molecular subtypes of colorectal cancer. Nat Med 2015;21:1350-6.

5. Kang H, O'Connell JB, Maggard MA, Sack J, Ko CY. A 10-year outcomes evaluation of mucinous and signet-ring cell carcinoma of the colon and rectum. Dis Colon Rectum 2005;48:1161-8.

6. Inamura K, Yamauchi M, Nishihara R, Kim SA, Mima K, Sukawa $\mathrm{Y}$, et al. Prognostic significance and molecular features of signetring cell and mucinous components in colorectal carcinoma. Ann Surg Oncol 2015;22:1226-35.

7. Jung SH, Kim SH, Kim JH. Prognostic impact of microsatellite instability in colorectal cancer presenting with mucinous, signetring, and poorly differentiated cells. Ann Coloproctol 2016;32: 58-65.

8. Le DT, Uram JN, Wang H, Bartlett BR, Kemberling H, Eyring $\mathrm{AD}$, et al. PD-1 blockade in tumors with mismatch-repair deficiency. N Engl J Med 2015;372:2509-20.

9. Meng X, Huang Z, Teng F, Xing L, Yu J. Predictive biomarkers in PD-1/PD-L1 checkpoint blockade immunotherapy. Cancer Treat Rev 2015;41:868-76.

10. Dudley JC, Lin MT, Le DT, Eshleman JR. Microsatellite Instability as a Biomarker for PD-1 Blockade. Clin Cancer Res 2016;22:81320. 